\title{
High temperature Bose-Einstein condensation
}

\author{
Viktor Begun ${ }^{1, a}$ \\ ${ }^{1}$ Institute of Physics, Jan Kochanowski University, Kielce, Poland
}

\begin{abstract}
The indications of a possible pion condensation at the LHC are summarized. The condensation is predicted by the non-equilibrium hadronization model for $2.76 \mathrm{TeV}$ $\mathrm{Pb}+\mathrm{Pb}$ collisions at the LHC. The model solves the proton/pion puzzle and reproduces the low $p_{T}$ enhancement of the pion spectra, as well as the spectra of protons and antiprotons, charged kaons, $K_{S}^{0}, K^{*}(892)^{0}$ and $\phi(1020)$. The obtained parameters allow to estimate the amount of pion condensate on the level of $5 \%$ from the total number of pions at the LHC. The condensate is located at $p_{T}<250 \mathrm{MeV}$.
\end{abstract}

\section{Introduction}

Bose-Einstein condensation (BEC) was predicted right after the introduction of Bose statistics [1]. However, it took 70 years to observe BEC experimentally [2]. The main difficulty for obtaining BEC in a gas of atoms is that they form a liquid or a solid before reaching BEC. It can be avoided, if extremely low densities and temperatures are achieved. This was done only after the development of the corresponding technologies, and the leaders of the two groups that created the condensate received the Nobel Prize in 2001 [3]. It might be the reason for the widespread delusion that BEC means low temperatures and atomic gases only. However, all conventional two-quark mesons are bosons and may condense. The temperatures that are reached in high-energy collision are of the MeV scale. It corresponds to $\sim 10^{10}$ Kelvin and is far above the BEC temperatures for cold atoms. The ratio of the BEC temperature in atomic gases, $T_{C}(A)$, to that in the pion gas, $T_{C}(\pi)$, is even larger, because one should take into account a possible size of the system and the masses of the particles. One obtains $10^{12}$ times higher temperature for the gas of pions [4]:

$$
\frac{T_{C}(\pi)}{T_{C}(A)} \simeq \frac{m_{A}}{m_{\pi}}\left(\frac{r_{A}}{r_{\pi}}\right)^{2} \simeq \frac{m_{A}}{m_{\pi}} 10^{10} \simeq 10^{12}
$$

Besides of much higher temperature, the properties of a BEC of mesons could be very different from the low-temperature BEC of atoms. The most obvious differences are the much smaller volumes, much higher densities, and different interaction forces involved in the formation of the high temperature BEC. The clear advantage of the pion BEC is that pion system is in the form of gas at freeze-out. The reason to consider pion $\mathrm{BEC}$ now is the recent data from $\mathrm{Pb}+\mathrm{Pb}$ collisions at $\sqrt{s_{N N}}=2.76 \mathrm{TeV}$ energies at Large Hadron Collider (LHC), that contain the set of puzzles.

\footnotetext{
a e-mail: viktor.begun@gmail.com
} 
- The predictions of the hadron-resonance gas (HRG) were too high for ratios to pions, especially for proton to pion ratio [5].

- The best fit of the LHC data by the HRG still gives nearly three standard deviations for protons [6].

- The low-transverse-momentum pion spectra show up to $50 \%$ enhancement compared to hydrodynamic models [5].

- The temperature obtained in HRG at the LHC falls out from the freeze-out line deeply in the hadronic phase [7].

The HRG worked well for smaller collisions energies. Pions and protons are among the most abundant particles. Therefore the problems with their description look suspicious. There are several solution proposed to explain the proton to pion ratio. The hadronization and freeze-out in a chemical non-equilibrium [8]. The separate freeze-out for strange particles [9]. An incomplete list of hadrons [10], and hadronic rescattering in the final stage [11]. However, none of them is commonly accepted yet.

There are deep physical reasons for the non-equilibrium and pion condensation at the LHC. It can be due to fast expansion and overcooling of the QGP [12, 13], or due to gluon condensation in Color Glass Condensate [14], and subsequent hadronization of the low $p_{T}$ gluons into low $p_{T}$ pions [15]. The non-equilibrium hadronization can explain the measured particle ratios [8], and also the spectra $[16,17]$ very well. The obtained parameters indicate the possibility that $5 \%$ of the total number of pions can be in BEC at the LHC [18]. The calculations of the pion spectra with the assumption of BEC show that the maximal transverse momentum that the BEC can obtain due to the expansion of the fireball is $p_{T}<250 \mathrm{MeV}$ [18]. It is the same region where ALICE Collaboration observes the emission of $23 \%$ of pions from a coherent source [19].

\section{The non-equilibrium model}

The thermodynamic motivation behind the non-equilibrium HRG (NEQ) is the following. The fast expansion of the fireball may cause it's overcooling, so that the number of quarks and anti-quarks is larger than their equilibrium value at the reached temperature. Therefore, the subsequent freezeout may lead to the formation of mesons and baryons with the multiplicities that are higher than the corresponding equilibrium numbers at the given temperature. The phase-space distribution of the primordial particles in NEQ is similar to the usual HRG:

$$
f_{i}=g_{i} \int \frac{d^{3} p}{(2 \pi)^{3}} \frac{1}{\gamma_{i}^{-1} \exp \left(\sqrt{p^{2}+m^{2}} / T\right) \pm 1},
$$

where $g_{i}$ is the degeneracy factor, $p$-momentum, $m$-mass, $T$-temperature, and

$$
\gamma_{i}=\gamma_{q}^{N_{q}^{i}+N_{\bar{q}}^{i}} \gamma_{s}^{N_{s}^{i}+N_{s}^{i}} \exp \left(\frac{\mu_{B} B_{i}+\mu_{Q} Q_{i}+\mu_{S} S_{i}}{T}\right)
$$

The $\gamma_{i}$ contains the chemical potentials and corresponding conserved charges $(Q, B, S)$ of a particle $i$ in equilibrium HRG (EQ), and the non-equilibrium parameters $\gamma_{q}, \gamma_{s}$. The $N_{q}^{i}, N_{\bar{q}}^{i}$ and $N_{s}^{i}, N_{\bar{s}}^{i}$ are the numbers of light $(u, d)$ and strange $(s)$ quarks and anti-quarks in the $i$-th hadron, correspondingly. Note the plus sign between the number of quarks and anti-quarks, in contrast to the usual conserved charges, that are defined as the difference between particles and anti-particles. One can exponentiate $\gamma_{q}$ and $\gamma_{s}$ and see that each particle in NEQ obtains it's own chemical potential, which can be also recalculated into a different temperature. Therefore NEQ is a particular case of the multiple-freeze out 
model, where each particle has it's own freeze-out, but the relation between them is fixed by Eq. (3). The NEQ is advocated by J. Rafelski and collaborators since a long time, see Ref. [20] for the review. It is implemented in the SHARE model [21] and allows for the HRG fit of the particle multiplicities and ratios. The THERMINATOR Monte-Carlo event generator [22] inherits the same particle and decay list and allows for further calculation of the particle spectra from the Cooper-Frye formula at the freeze-out hypersurface $\Sigma_{\mu}$ :

$$
\frac{d N}{d y d^{2} p_{T}}=\int d \Sigma_{\mu} p^{\mu} f(p \cdot u), \quad t^{2}=\tau_{f}^{2}+x^{2}+y^{2}+z^{2}, \quad x^{2}+y^{2} \leq r_{\max }^{2},
$$

where $\tau_{f}$ and $r_{\max }$ are the characteristic time and radius of the hypersurface. There are several hypersurfaces implemented in THERMINATOR following the paper [23]. For the LHC we use Cracow model, that assumes the Hubble-like flow of particles: $u^{\mu}=x^{\mu} / \tau_{f}$, because it fits the data the best. At the LHC $\mu_{B} \simeq \mu_{Q} \simeq \mu_{S} \simeq 0$ due to large collision energy compared to the rest mass of the colliding nuclei. Therefore, EQ model has two parameters left - temperature and volume, while NEQ has also $\gamma_{q}$ and $\gamma_{s}$. The measured set of particles, that was used in [16-18, 24], includes 6 to 8 multiplicities depending on centrality. It allows to determine both the parameters in EQ and in NEQ.

There is only one additional parameter in the model that allows to describe the spectra, because the product $\pi \tau_{f} r_{\max }^{2}$ is equal to the volume per unit rapidity, while the ratio $r_{\max } / \tau_{f}$ determines the slopes of the spectra. The procedure is the following: first, the volume and temperature are found from the fit of all mean multiplicities, and then the $r_{\max } / \tau_{f}$ is found from the best fit to the spectra of pions $\pi^{+}+\pi^{-}$ and kaons $K^{+}+K^{-}$. This approach gives unexpectedly good results in NEQ. The fit that was made for the spectra of pions and kaons only, appears also very good for $p+\bar{p}, K_{S}^{0}, K^{*}(892)^{0}$ and $\phi(1020)$, see Figs. 1, 2. The temperature in EQ is much higher, therefore the amount of secondary pions is larger
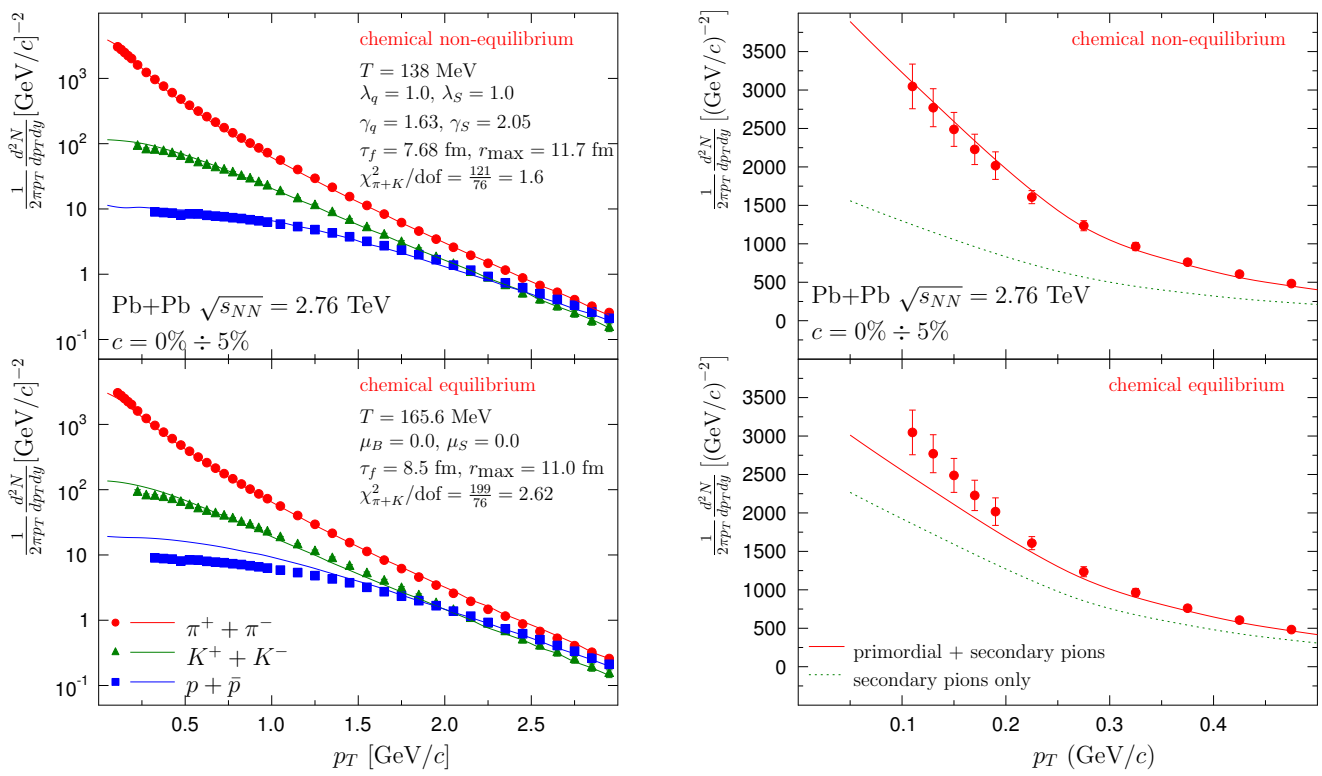

Figure 1. The spectra of pions, kaons and protons (left) in NEQ (up) and EQ (down), and the same only for pions in linear scale (right). The figures are from Ref. [17]. 


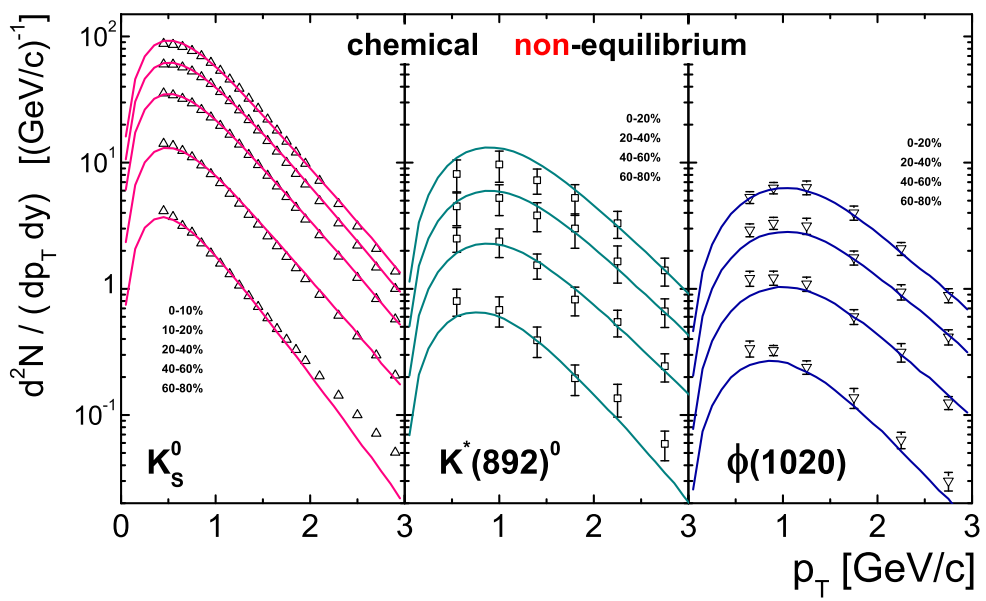

Figure 2. The spectra of strange particles in NEQ. The figure is from Ref. [16].

in NEQ. However, it is not enough to reproduce the steepness of the spectrum at $p_{T}<250 \mathrm{MeV}$, see Fig. 1 (right). The best state of the art fit of pion, kaon and proton spectra in EQ allows to describe protons taking into account rescattering mechanism [25]. Unfortunately, the authors of Ref. [25] do not provide the plot for pions in a linear scale, or the data/model ratio. However, one can see that they still have about $30 \%$ deficit of pions in the model at $p_{T}<250 \mathrm{MeV}$. The simultaneous fit of $K^{*}(892)^{0}$ and $\phi(1020)$ in NEQ is as surprising, as the applicability of the pion-kaon fit to protons. The $K^{*}(892)^{0}$ is short living, while $\phi(1020)$ is long living. They should feel a long rescattering phase differently [26]. The successful simultaneous description of protons, pions, $K^{*}(892)^{0}$ and $\phi(1020)$ in NEQ without rescattering may indicate that the rescattering phase is well parameterized by the non-equilibrium parameters (3).

The numerical value of the $\gamma_{q}$ recalculated into the corresponding chemical potential is very close to the pion mass: $\mu_{\pi}=2 T \ln \gamma_{q} \simeq 134 \mathrm{MeV} \simeq m_{\pi^{0}} \simeq 134.98 \mathrm{MeV}$. It suggests that a substantial part of $\pi^{0}$ mesons tend to form the condensate, but this possibility is not allowed in the HRG implementation in SHARE and THERMINATOR. It can be done by explicit treatment of hadronic ground states. When chemical potential approaches the mass of a particle, $\mu \rightarrow m$, the discrete sum over the low momentum quantum levels should be taken, instead of the integral over momentum. It can be shown that in the infinite volume limit, $V \rightarrow \infty$, only the zero momentum level, $p_{0}=0$, can be taken into account [4]. Then the total mean multiplicity of a particle, $N_{\text {tot }}$, receives one more term:

$$
\begin{aligned}
N_{\text {tot }} & =\sum_{j} \frac{1}{\exp \left[\left(\sqrt{p_{j}^{2}+m^{2}}-\mu\right) / T\right]-1} \\
& \simeq \frac{1}{\exp [(m-\mu) / T]-1}+V \int_{0}^{\infty} \frac{d^{3} p}{(2 \pi)^{3}} \frac{1}{\exp \left[\left(\sqrt{p^{2}+m^{2}}-\mu\right) / T\right]-1} \\
& =N_{\text {cond }}+N_{\text {norm }},
\end{aligned}
$$

where $N_{\text {cond }}$ is the number of particles in the condensate. When chemical potential approaches the mass, the $N_{\text {cond }}$ grows infinitely and competes with arbitrarily large volume, therefore the $\gamma_{q}$ does not grow so much. The corresponding changes were introduced into SHARE. The new fit in the NEQ 
model with explicit treatment of the ground state $(\mathrm{BEC})^{1}$ gives rather different results, see Fig. 3 . The inclusion of the ground state makes equilibrium and non-equilibrium models closer. They even
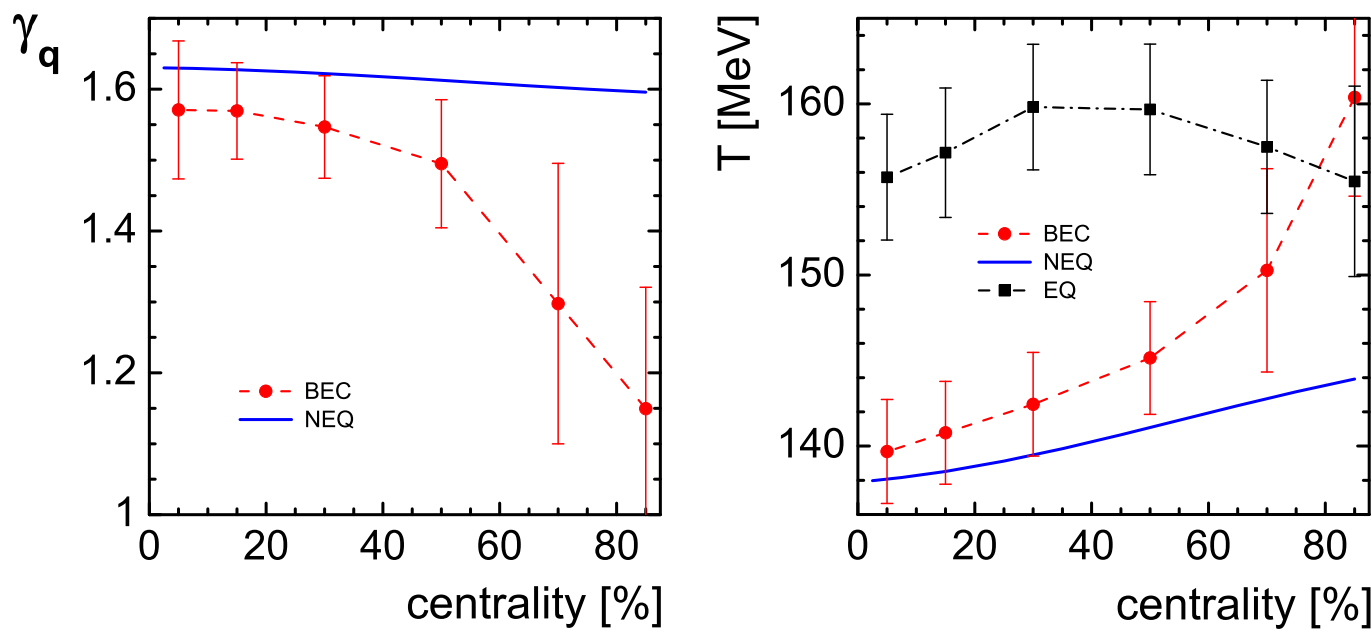

Figure 3. The non-equilibrium parameter $\gamma_{q}$ (left) and temperature (right) as the function of centrality. The figures are from Ref. [24]

coincide at high centrality, because $\gamma_{q}^{\mathrm{BEC}} \simeq 1$ there, while $\gamma_{q}^{\mathrm{EQ}}=1$ by definition.

Pion spectra with the condensate were calculated in Ref. [18] using the appropriately modified THERMINATOR. The condensate is at rest only in it's reference frame and is moving with the freeze-out hypersurface. The approximation (5) leads to the step at $p_{T} \sim 230 \mathrm{MeV}$, because the corresponding momentum distribution is given by:

$$
\frac{d N}{d y d \phi_{p} p_{T} d p_{T}}=\frac{N_{\text {cond }}}{V} \frac{\tau_{f}^{3}}{m^{2}} \theta\left(r_{\max }-p_{T} \tau_{f} / m\right) .
$$

where $\theta$ is the Heaviside step function. The inclusion of several more low lying levels would lead to finer steps that tend to a continuous line with increasing number of steps, and have the same area under the curve. The current approximation shows the maximal momentum that the condensate may obtain from the movement of the freeze-out hypersurface, which is $p_{T}<250 \mathrm{MeV}$.

The condensate rate grows with centrality in BEC, but the similar growths shows EQ model, see Fig. 4 (left). It is the finite volume effect, which meas that the ground state $N_{0}^{E Q}=N_{\text {cond }}(\mu=0)$ should be taken into account even in equilibrium for very peripheral, proton-proton, or proton-nucleus collisions. The gray area shows the $10 \%$ deviation from the best fit. It is well within the error bars at central, but not in peripheral collisions, see Fig. 4 (right). The combined data on multiplicities and spectra are compatible with $5 \%$ of pions in the condensate [18].

The recent PDG reviews [27] report much lower mass and width of the $f_{0}(500)$ resonance, or the sigma meson $(\sigma)$, see Fig. 5, and Ref. [28, 29] for explanations. Lower mass results in higher multiplicity in HRG. The $\sigma$ meson decays $100 \%$ into pions, therefore the inclusion of the updated $\sigma$ could have added some of the missing pions and weaken the BEC signal. The Breit-Wigner distribution

\footnotetext{
${ }^{1}$ The same abbreviation BEC is used for the Bose-Einstein condensation and for the non-equilibrium model that allows the condensation on the ground state.
} 

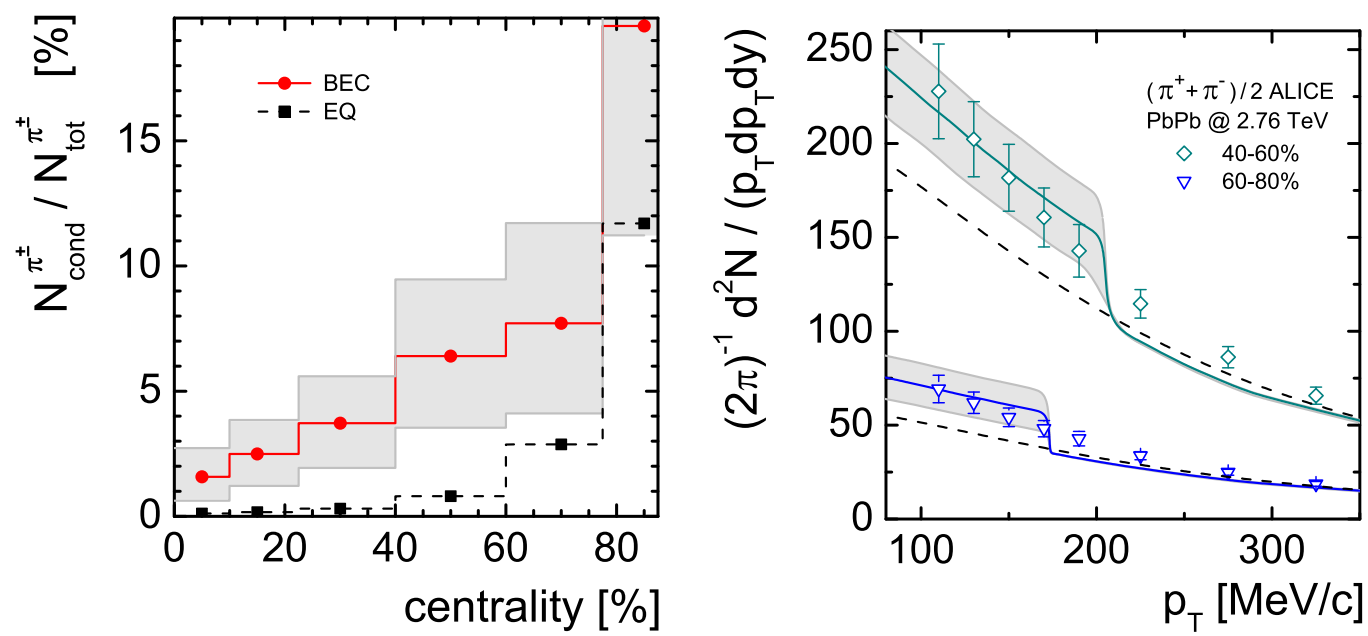

Figure 4. The pion spectra (left) and the condensate rate that was found in BEC (right) by the fit of mean multiplicities at the LHC. Solid line is for BEC, while dashed line is for EQ. The figures are from Ref. [18].
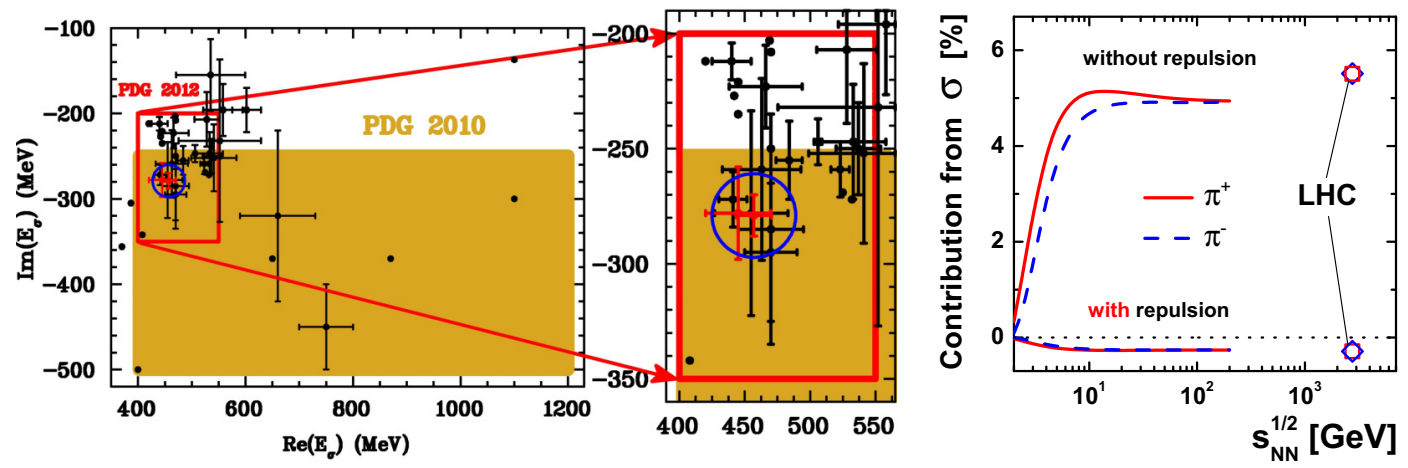

Figure 5. The change of the width and mass of the $\sigma$ meson since 2010 (left), and the contribution to pion yields from the decay of $\sigma$ (right). The left figure is from Ref. [30].

is not accurate enough for so wide resonances as $\sigma$. However, the width of the $\sigma$ can be obtained from the derivative of the experimental $\pi \pi$ phase shift, that we take from [28]. It has attractive $(0 ; 0)$ and repulsive $(2 ; 0)$ isospin-spin channel. The attractive one is responsible for the emergence of the $f_{0}(500)$ resonance, however, the repulsive one cancels $f_{0}(500)$ until $f_{0}(980)$ takes over above the mass $M_{\pi \pi} \sim 0.85 \mathrm{GeV}$, see Ref. [31]. The cancellation happens on the level of the distribution function, therefore it is present in all isospin-averaged observables. The $\sigma$ implemented as a Breit-Winger pole with $M_{\sigma}=484$ and $\Gamma_{\sigma} / 2=255 \mathrm{MeV}$ produces up to $5 \%$ of pions, while the truth contribution is $-0.3 \%$, see Fig. 5 (right) [31]. The absence of pions from the $\sigma$ decays enhances all ratios to pions, compared to the results that were obtained in models with $\sigma$. It is particularly important for the description of the $K / \pi$ horn at the SPS $[32,33]$ and the proton-pion puzzle at the LHC. 


\section{Conclusions}

The non-equilibrium thermal model combined with the single freeze-out scenario explains very well the spectra of $\pi, K, p, K_{S}^{0}, K^{*}(892)^{0}$ and $\phi(1020)$ particles at the LHC. The introduction of the ground state decreases the non-equilibrium parameters and increases the temperature with centrality. The enhancement of the low $p_{T}$ pion spectra may be interpreted as a signature of the onset of pion condensation at the LHC. The missing pions from the $\sigma$ meson in HRG enhance the proton-pion puzzle at the LHC, and allow for larger amount of the condensate. Many efforts and attention of the community is required to obtain high temperature BEC and study it's properties. However, it could open a wide new field of research.

\section{Acknowledgments}

The author is thankful to W. Florkowski, M. Rybczynski, M. Gorenstein, W. Broniowski and F. Giacosa for fruitful collaboration. This work was supported by Polish National Science Center grant No. DEC-2012/06/A/ST2/00390.

\section{References}

[1] S. N. Bose, Z. Phys. 26, 178 (1924); A. Einstein, Sitz. Ber. Preuss. Akad. Wiss. (Berlin) 1, 3 (1925).

[2] M. H. Anderson, et al., Science 269, 198 (1995); K. B. Davis, et al., Phys. Rev. Lett. 75, 3969 (1995).

[3] Editor Tore Frangsmyr, Les Prix Nobel, (The Nobel Prizes 2001, Nobel Foundation, Stockholm, 2002) $77-108$.

[4] V. V. Begun and M. I. Gorenstein, Phys. Rev. C 77, 064903 (2008) [arXiv:0802.3349 [hep-ph]].

[5] B. Abelev et al. [ALICE Collaboration], Phys. Rev. C 88, 044910 (2013) [arXiv:1303.0737 [hepex]].

[6] J. Stachel, A. Andronic, P. Braun-Munzinger and K. Redlich, J. Phys. Conf. Ser. 509, 012019 (2014) doi:10.1088/1742-6596/509/1/012019 [arXiv:1311.4662 [nucl-th]].

[7] J. Cleymans, EPJ Web Conf. 95, 03004 (2015) [arXiv:1412.7045 [hep-ph]].

[8] M. Petran, J. Letessier, V. Petracek and J. Rafelski, Phys. Rev. C 88, no. 3, 034907 (2013) [arXiv:1303.2098 [hep-ph]].

[9] S. Chatterjee, B. Mohanty and R. Singh, Phys. Rev. C 92, no. 2, 024917 (2015) [arXiv:1411.1718 [nucl-th]].

[10] J. Noronha-Hostler and C. Greiner, Nucl. Phys. A 931, 1108 (2014) [arXiv:1408.0761 [nucl-th]].

[11] F. Becattini, M. Bleicher, T. Kollegger, T. Schuster, J. Steinheimer and R. Stock, Phys. Rev. Lett. 111, 082302 (2013) [arXiv:1212.2431 [nucl-th]].

[12] E. Shuryak, arXiv:1412.8393 [hep-ph].

[13] T. Csorgo and L. P. Csernai, Phys. Lett. B 333, 494 (1994) [hep-ph/9406365].

[14] J. P. Blaizot, F. Gelis, J. F. Liao, L. McLerran and R. Venugopalan, Nucl. Phys. A 873, 68 (2012) [arXiv:1107.5296 [hep-ph]].

[15] F. Gelis, Nucl. Phys. A 931, 73 (2014) [arXiv:1412.0471 [hep-ph]].

[16] V. Begun, W. Florkowski and M. Rybczynski, Phys. Rev. C 90, no. 5, 054912 (2014) [arXiv:1405.7252 [hep-ph]].

[17] V. Begun, W. Florkowski and M. Rybczynski, Phys. Rev. C 90, no. 1, 014906 (2014) [arXiv:1312.1487 [nucl-th]]. 
[18] V. Begun and W. Florkowski, Phys. Rev. C 91, 054909 (2015) [arXiv:1503.04040 [nucl-th]].

[19] B. B. Abelev et al. [ALICE Collaboration], Phys. Rev. C 89, no. 2, 024911 (2014) [arXiv:1310.7808 [nucl-ex]].

[20] J. Rafelski, Eur. Phys. J. A 51, no. 9, 114 (2015) [arXiv:1508.03260 [nucl-th]].

[21] G. Torrieri, S. Steinke, W. Broniowski, W. Florkowski, J. Letessier and J. Rafelski, Comput. Phys. Commun. 167, 229 (2005) [nucl-th/0404083].

[22] M. Chojnacki, A. Kisiel, W. Florkowski and W. Broniowski, Comput. Phys. Commun. 183, 746 (2012) [arXiv:1102.0273 [nucl-th]].

[23] W. Broniowski and W. Florkowski, Phys. Rev. Lett. 87, 272302 (2001) [nucl-th/0106050].

[24] V. Begun, EPJ Web Conf. 97, 00003 (2015) [arXiv:1412.6532 [nucl-th]].

[25] S. Ryu, J.-F. Paquet, C. Shen, G. S. Denicol, B. Schenke, S. Jeon and C. Gale, Phys. Rev. Lett. 115, no. 13, 132301 (2015) [arXiv:1502.01675 [nucl-th]].

[26] A. G. Knospe, C. Markert, K. Werner, J. Steinheimer and M. Bleicher, arXiv:1509.07895 [nuclth].

[27] K. A. Olive et al. [Particle Data Group Collaboration], Chin. Phys. C 38, 090001 (2014).

[28] R. Garcia-Martin, R. Kaminski, J. R. Pelaez and J. Ruiz de Elvira, Phys. Rev. Lett. 107, 072001 (2011) [arXiv:1107.1635 [hep-ph]].

[29] J. R. Pelaez, arXiv:1510.00653 [hep-ph].

[30] R. Kaminski, Acta Phys. Polon. Supp. 8, no. 1, 103 (2015).

[31] W. Broniowski, F. Giacosa and V. Begun, Phys. Rev. C 92, no. 3, 034905 (2015) [arXiv:1506.01260 [nucl-th]].

[32] M. Gazdzicki and M. I. Gorenstein, Acta Phys. Polon. B 30, 2705 (1999) [hep-ph/9803462].

[33] C. Alt et al. [NA49 Collaboration], Phys. Rev. C 77, 024903 (2008) [arXiv:0710.0118 [nucl-ex]]. 